\section{Additive Thoraxbestrahlung verlängert das Überleben}

\author{
Die Prognose für Patienten mit kleinzelligem Bronchialkarzinom \\ (SCLC) im extensiven Stadium ist äußerst schlecht. Sprechen \\ Patienten auf die übliche Chemotherapie mit Cisplatin plus \\ Etoposid an, kann mit einer Ganzhirn-Bestrahlung ein \\ Überlebensvorteil erreicht werden. Ein Problem bleibt die \\ intrathorakale Tumorkontrolle. Daher haben B. J. Slotmann et \\ al. den Effekt einer zusätzlichen Thoraxbestrahlung analysiert. \\ Lancet 2015; 385: 36-42
}

An der Phase-III-Studie waren 42 Kliniken in den Niederlanden, in Großbritannien, in Norwegen und Belgien beteiligt. Teilnehmen konnten Patienten im Alter von über 18 Jahren mit SCLC im extensiven Stadium, die auf 4-6 Zyklen StandardChemotherapie angesprochen hatten. Spätestens 7 Wochen nach der Chemotherapie erhielten die Teilnehmer randomisiert entweder nur eine prophylaktische Ganzhirn- oder zusätzlich eine Thoraxbestrahlung.

Die Bestrahlung des Brustkorbs umfasste 10 Fraktionen (Gesamtdosis $30 \mathrm{~Gy}$ ) mit einem Sicherheitssaum von 15 mm zum Tumorvolumen nach Chemotherapie, wobei Lymphknoten im Mediastinum ebenfalls bestrahlt wurden. Primärer Endpunkt war das Gesamtüberleben nach einem

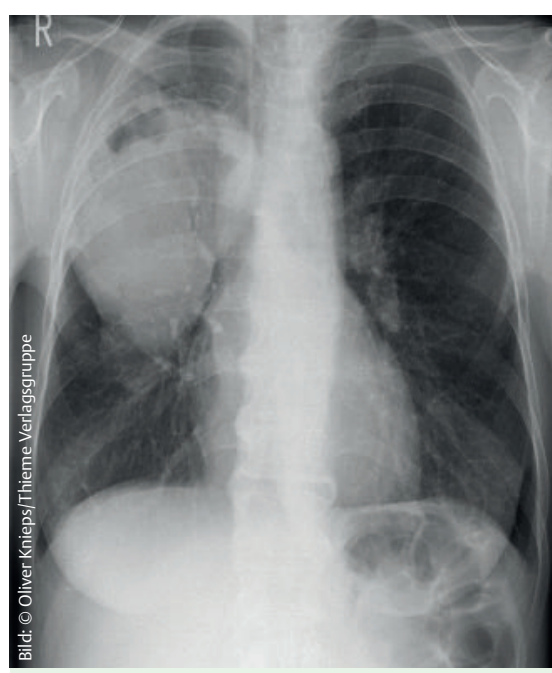

Die 2-Jahres-Überlebensraten können beim SCLC unter $5 \%$ liegen. Hier ein Bronchialkarzinom mit unbekanntem Subtyp.
Jahr. Zusätzlich wurde das Gesamtüberleben nach 2 Jahren ausgewertet. Zu den sekundären Endpunkten zählten die intrathorakale Tumorkontrolle, das progressionsfreie Überleben (median und nach 6 Monaten) sowie toxische Effekte.

\section{2-Jahres-Überleben verbessert \\ $\nabla$}

Von den 495 Teilnehmern waren $23 \%$ über 70 und $8 \%$ über 75 Jahre alt. Insgesamt 247 Patienten erhielten die additive Thoraxbestrahlung und 248 die alleinige Ganzhirnbestrahlung (Kontrollgruppe). Zwischen Diagnose und Randomisierung lagen im Schnitt 17 Wochen, die mediane Nachbeobachtungszeit betrug 24 Monate. In beiden Gruppen war das 1-Jahresüberleben vergleichbar ( 33 vs. $28 \%, p=0,066$ ) und das mediane Gesamtüberleben erreichte 8 Monate. Nach 18 Monaten überlebten in der Gruppe mit Thoraxbestrahlung 16\% und in der Kontrollgruppe 9\% $(\mathrm{p}=0,03)$. Die 2-Jahres-Überlebensrate ergab mit 13\% (Thoraxbestrahlung) vs. 3\% (Kontrollgruppe) einen statistisch signifikanten Unterschied von $\mathrm{p}=0,004$; um einen Todesfall zu verhindern, mussten 10,6 Patienten behandelt werden.

Die additive Thoraxbestrahlung reduzierte das Progressionsrisiko um 27\% (Hazard Ratio [HR] 0,73, p=0,001). Nach 6 Monaten Thoraxbestrahlung waren $24 \%$ progressionsfrei, in der Kontrollgruppe waren es $20 \%(p=0,001)$. Es wurden keine schweren toxischen Effekte beobachtet; zu den häufigsten Nebenwirkungen gehörten in beiden Gruppen gleichermaßen Fatigue und Dyspnoe.

\section{Fazit}

Wie die vorliegende Studie zeigt, kann bei SCLC-Patienten im extensiven Stadium die additive Thoraxbestrahlung das 2-Jahres-Überleben deutlich verbessern. Gleichzeitig wurde das progressionsfreie Überleben verbessert. Nach Meinung der Autoren sollte die Thoraxbestrahlung bei diesen Patienten nach Ansprechen auf eine Chemotherapie in Erwägung gezogen werden.

Matthias Manych, Berlin

\section{Tuberkulose}

\section{Informationsbericht des DZK 2015}

Das Deutsche Zentralkomitee zur Bekämpfung der Tuberkulose e.V. (DZK) veröffentlicht im August seinen 38. Informationsbericht. Der diesjährige Bericht enthält wieder aktuelle Beiträge zu den wichtigen Themen, Entwicklungen und neuen Erkenntnissen hinsichtlich der Tuberkulose(Situation) in Deutschland und im Ausland.

Neben Informationen zur Epidemiologie in Deutschland, Europa und weltweit wird sich der diesjährige Informationsbericht u.a. dem Thema „Tuberkulose und Migration in Deutschland“ widmen. Weiterhin gibt es dieses Jahr interessante Beiträge zu den neuen Empfehlungen der Weltgesundheitsorganisation (WHO) zur latenten Tuberkulose-Infektion und einen Beitrag zu nicht tuberkulösen Mykobakterien. Außerdem wird die Arbeit des Stop-Tb-Forums und der Günther-LabesStiftung vorgestellt und über nationale und internationale Kongresse berichtet.

Der 38. Informationsbericht des DZK 2015 hält für Interessierte eine informative Übersicht zu aktuellen Themen und Fragen der Tuberkulosebekämpfung im In- und Ausland bereit. Er kann zum Preis von $15 €$ zzgl. Porto $+7 \%$ MwSt. beim DZK bestellt werden. Weitere Informationen finden Sie online unter www.pneumologie.de/dzk

Nach einer Mitteilung des DZK, Berlin 